\title{
The natural history of cerebral cavernous malformations in children
}

\author{
Bradley A. Gross, MD, ${ }^{1-4}$ Rose Du, MD, PhD, ${ }^{1,4}$ Darren B. Orbach, MD, PhD, ${ }^{3,4}$ \\ R. Michael Scott, MD, ${ }^{2,4}$ and Edward R. Smith, MD2,4 \\ 1Department of Neurological Surgery, Brigham and Women's Hospital; 2Department of Neurological Surgery; and 'Division of \\ Neurointerventional Radiology, Boston Children's Hospital, ${ }^{4}$ Harvard Medical School, Boston, Massachusetts
}

OBJECTIVE Cerebral cavernous malformations (CMs) are a source of neurological morbidity from seizures and focal neurological deficits due to mass effect and hemorrhage. Although several natural history reports exist for adults with CMs, similar data for pediatric patients are limited.

METHODS The authors reviewed hospital databases to identify children with CMs who had not been treated surgically and who had clinical and radiological follow-up. Annual hemorrhage rates were calculated in lesion-years, and risk factors were assessed using the Cox proportional hazards model.

RESULTS In a cohort of 167 patients with $222 \mathrm{CMs}$, the mean patient age at the time of diagnosis was 10.1 years old (SD 6.0). Ninety patients (54\%) were male. One hundred four patients (62\%) presented with hemorrhage from at least 1 CM, 58 (35\%) with seizures with or without CM hemorrhage, and $43(26 \%)$ with incidental lesions. Twenty-five patients $(15 \%)$ had multiple CMs, $17(10 \%)$ had a family history of CMs, and $33(20 \%)$ had radiologically apparent developmental venous anomalies (DVAs). The overall annual hemorrhage rate was 3.3\%. Permanent neurological morbidity was $29 \%$ per hemorrhage, increasing to $45 \%$ for brainstem, thalamic, or basal ganglia CM and decreasing to $15 \%$ for supratentorial lobar or cerebellar lesions. The annual hemorrhage rate for incidental CMs was $0.5 \%$; for hemorrhagic $\mathrm{CMs}$, it was $11.3 \%$, increasing to $18.2 \%$ within the first 3 years. Hemorrhage clustering within 3 years was statistically significant (HR $6.1,95 \% \mathrm{Cl} 1.72-21.7, \mathrm{p}=0.005)$. On multivariate analysis, hemorrhagic presentation (HR 4.63, 95\% Cl 1.53-14.1, $p=$ 0.007 ), brainstem location (HR 4.42,95\% Cl 1.57-12.4, $p=0.005$ ), and an associated radiologically apparent DVA (HR 2.91, 95\% Cl 1.04-8.09, $p=0.04$ ) emerged as significant risk factors for hemorrhage, whereas age, sex, CM multiplicity, and CM family history did not.

CONCLUSIONS Prior hemorrhage, brainstem location, and associated DVAs are significant risk factors for symptomatic hemorrhage in children with CMs. Hemorrhage clustering within the first 3 years of a bleed can occur, a potentially important factor in patient management and counseling.

http://thejns.org/doi/abs/10.3171/2015.2.PEDS14541

KEY WORDS cavernous malformation; cavernoma; pediatric; children; hemorrhage; seizure; natural history; vascular disorders

$\mathrm{C}$ EREBRAL cavernous malformations (CMs) may follow a dynamic course including lesion enlargement,

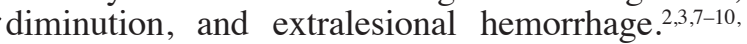
12,14,21-23 The literature evaluating the natural history of CMs in the pediatric population is limited. ${ }^{2,13}$ The purpose of this report is to elucidate the natural history of newly diagnosed CMs in the pediatric population to determine their eventual clinical course, with an emphasis on risk factors for hemorrhage.

\section{Methods}

\section{Population and Inclusion Criteria}

With the approval of the Boston Children's Hospital Institutional Review Board, we undertook a retrospective review of departmental and hospital databases to find all pediatric patients (21 years of age or younger) who had been diagnosed with cerebral CM in the period from 1997 through 2012. We required that all lesions be at least $4 \mathrm{~mm}$

ABBREVIATIONS CM = cavernous malformation; DVA = developmental venous anomaly.

ACCOMPANYING EDITORIAL See pp 121-122. DOI: 10.3171/2015.4.PEDS15115.

SUBMITTED October 6, 2014. ACCEPTED February 20, 2015.

INCLUDE WHEN CITING Published online October 16, 2015; DOI: 10.3171/2015.2.PEDS14541. 
in size and visible on T2-weighted MRI for inclusion in our analysis; those visible only on susceptibility-weighted imaging were excluded.

\section{Data Collection and Analysis}

We reviewed patient charts to determine the following information: age at presentation, sex, modality of presentation, $\mathrm{CM}$ location, CM multiplicity, CM family history, history of radiation, and associated developmental venous anomaly (DVA). The time of initial clinical and radiological (MRI) diagnosis was noted, as was the time of the last clinical and radiological follow-up. The development of any new T2-visible CMs at least $4 \mathrm{~mm}$ in size at the radiological follow-up was also recorded.

To be included in our analysis of annual hemorrhage risk, patients had to have at least 1 month of untreated follow-up; this included patients ultimately undergoing resection of their CM. Follow-up was tallied in lesion-years from the time of initial presentation until the last clinical follow-up or initiation of treatment. Hemorrhage was defined as the presence of new acute bleeding on imaging, with an associated new neurological deficit, seizure, and/ or apoplectic presentation. Annual hemorrhage rates were calculated as the ratio of the number of bleeds to the total number of lesion-years of follow-up.

\section{Statistical Analysis}

For our analysis of hemorrhage risk, the primary outcome measure was symptomatic hemorrhage. Time 0 was defined as age at presentation, and the first hemorrhage after presentation was used as the censoring event (right censoring). To explain the variability in the primary outcome, we examined 7 variables per lesion: age, sex, presentation modality (seizure, hemorrhage), location (brainstem vs nonbrainstem), CM family history, CM multiplicity, and associated DVAs. The Survival package in R, version 2.14, was then used to calculate hazard ratios using the univariate and multivariate Cox proportional hazards regression models. A p value $<0.05$ was considered significant.

\section{Results}

\section{Background and Demographics}

In this cohort of 167 patients with $222 \mathrm{CMs}$, the mean patient age at the time of diagnosis was 10.1 years (SD 6.0, range $0.1-21$ years; Table 1$)$. Ninety patients $(54 \%)$ were male. One hundred four patients $(62 \%)$ presented with hemorrhage from at least $1 \mathrm{CM}, 58(35 \%)$ with seizures with or without CM hemorrhage, and $43(26 \%)$ with incidental lesions. Twenty-five patients (15\%) had multiple CMs, 17 (10\%) had a family history of CMs, and 33 (20\%) had radiologically apparent DVAs. One hundred seventyfour CMs were supratentorial lobar (78\%), 18 (8\%) were in the brainstem, $16(7 \%)$ were in the basal ganglia or thalamus, and $14(6 \%)$ were in the cerebellum. Interestingly, half (7 of 14) of the cerebellar CMs were associated with DVAs as compared with $21 \%$ of the brainstem CMs, $19 \%$ of the supratentorial deep CMs, and $15 \%$ of the supratentorial lobar CMs ( $\mathrm{p}=0.01$ for comparison of cerebellar CMs to all other locations by Fisher exact test).
TABLE 1. Summary of background and demographic information for 167 children with 222 cerebral CMs

\begin{tabular}{lc}
\hline \multicolumn{1}{c}{ Parameter } & No. \\
\hline Patients (CMs) & $167(222)$ \\
\hline Mean age in yrs (range) & $10.1(0.1-21)$ \\
\hline Male sex (\%) & $90(54)$ \\
\hline Presentation (\%) & \\
Incidental & $43(26)$ \\
Seizures (w/ or w/o hemorrhage) & $58(35)$ \\
Hemorrhage & $104(62)$ \\
\hline Radiologically apparent DVA (\%) & $33(20)$ \\
\hline Multiple CMs (\%) & $25(15)$ \\
\hline Family history of CMs (\%) & $17(10)$ \\
\hline Prior radiation (\%) & $15(9)$ \\
\hline CM location (\%) & \\
Supratentorial lobar & $174(78)$ \\
Supratentorial deep (basal ganglia/thalamus) & $16(7)$ \\
Cerebellar & $14(6)$ \\
Brainstem & $18(8)$ \\
\hline
\end{tabular}

\section{De Novo CM Development}

Over a total of 789.5 patient-years of radiological (MRI) follow-up from the time of an initial radiological diagnosis of at least $1 \mathrm{CM}, 20$ de novo CMs, each measuring at least $4 \mathrm{~mm}$ on T2-weighted MRI, were noted in 10 patients. This corresponds to an annual incidence of $2.5 \%$ per lesion or $1.2 \%$ per patient (Table 2). Six of these patients had initially presented with multiple CMs $(60 \%$; 3 had a known family history of CMs), and 2 had undergone prior radiation (20\%). Two patients had presented with solitary lesions: 1 was an infant with von Willebrand's disease who subsequently developed 2 de novo frontal lesions at the age of 4 years.

Twenty-five patients initially presenting with multiple CMs had 198.5 patient-years of radiological follow-up. Six of these patients developed 14 de novo CMs (measuring at least $4 \mathrm{~mm}$ on T2-weighted MRI), corresponding to an annual rate of $7.1 \%$ per lesion or $3.0 \%$ per patient (Table 2). Fifteen patients with radiation-induced CMs had 117 patient-years of radiological follow-up from the time of initial radiological diagnosis of their CMs. Two of these patients developed 3 de novo CMs at least $4 \mathrm{~mm}$ on T2-weighted imaging, corresponding to an annual rate of $2.6 \%$ per lesion or $1.7 \%$ per patient. Across the remaining group of patients presenting with single nonfamilial CMs without a prior history of radiation, the annual rate of de novo CM development was $0.6 \%$ per lesion or $0.4 \%$ per patient.

TABLE 2. Stratified annual rates of de novo CM development

\begin{tabular}{lcc}
\hline \multicolumn{1}{c}{ Group } & $\begin{array}{c}\text { Annual Rate } \\
\text { per Lesion }\end{array}$ & $\begin{array}{c}\text { Annual Rate } \\
\text { per Patient }\end{array}$ \\
\hline Overall & $2.5 \%$ & $1.2 \%$ \\
\hline Multiple CMs on presentation & $7.1 \%$ & $3.0 \%$ \\
\hline Prior radiation & $2.6 \%$ & $1.7 \%$ \\
\hline Solitary nonfamilial CM on presentation & $0.6 \%$ & $0.4 \%$ \\
\hline
\end{tabular}




\section{Radiation-Induced CMs}

Fifteen patients (9\%) had $23 \mathrm{CMs}$ that developed after prior radiation. Eight of these patients $(53 \%)$ were treated for acute lymphocytic leukemia, 4 (27\%) for medulloblastoma, and 1 each (7\%) for ependymoma, germinoma, and neuroblastoma. Mean patient age at the time of radiation was 5.2 years old (SD 2.3), and the mean latency until radiological diagnosis of the CM was 9.3 years (SD 4.8). There was no significant sex predilection in this cohort $(8$ males [53\%]). Seven patients (46\%) presented with hemorrhage and $4(27 \%)$ with seizures with or without hemorrhage. Seven patients $(46 \%)$ were initially asymptomatic despite their lesions. Cavernous malformation location was predominantly supratentorial lobar (21 [91\%] of 23); 1 lesion (4\%) was in the cerebellum and another (4\%) was in the basal ganglia. None of these CMs were associated with a DVA.

\section{Hemorrhage Rate and Morbidity}

One hundred fifty-nine patients with $193 \mathrm{CCMs}$ had at least 1 month of untreated follow-up and were included in our analysis of annual hemorrhage rate (follow-up range 0.1-16.4 years, mean 3.8 years). Over a total of 726.3 lesion-years, 24 hemorrhages occurred, corresponding to an overall annual hemorrhage rate of $3.3 \%$ per lesion-year (Table 3). For 73 incidental CMs, the annual hemorrhage rate was $0.5 \%$. Notably, $63(86 \%)$ of these incidental CMs had at least 1 year of untreated follow-up, and 36 (49\%) had at least 5 years of untreated follow-up. The annual hemorrhage rates in these 2 subgroups remained consistent at $0.5 \%$ and $0.3 \%$, respectively. For 43 patients presenting with solitary incidental CMs, the annual hemorrhage rate was $1.2 \%$.

The annual hemorrhage rate for hemorrhagic CMs was $11.3 \%$, increasing to $18.2 \%$ within the first 3 years of a $\mathrm{CM}$ hemorrhage. This rate decreased to $4.8 \%$ after 3 years

TABLE 3. Stratified annual hemorrhage rates*

\begin{tabular}{lcrc}
\hline \multicolumn{1}{c}{ CM Group } & Total Lesion-Years & Bleeds & Annual Rate \\
\hline Overall & 726.3 & 24 & $3.3 \%$ \\
\hline Female & 274.4 & 11 & $4.0 \%$ \\
Male & 451.9 & 13 & $2.9 \%$ \\
\hline Incidental & 428.7 & 2 & $0.5 \%$ \\
Headaches, no bleed & 54.2 & 2 & $3.7 \%$ \\
Epileptogenic, no bleed & 83.6 & 2 & $2.4 \%$ \\
Hemorrhagic & 159.8 & 18 & $11.3 \%$ \\
\hline Rebleed rate in first 3 yrs & 77.1 & 14 & $18.2 \%$ \\
Rebleed rate after 3 yrs & 82.7 & 4 & $4.8 \%$ \\
\hline Familial & 182.1 & 2 & $1.1 \%$ \\
Nonfamilial & 544.2 & 22 & $4.0 \%$ \\
\hline Superficial & 616.9 & 13 & $2.1 \%$ \\
Supratentorial Deep & 49.2 & 1 & $2.0 \%$ \\
Brainstem & 60.2 & 10 & $16.7 \%$ \\
\hline Multiplicity & 418.6 & 4 & $1.0 \%$ \\
Nonmultiplicity & 307.7 & 20 & $6.5 \%$ \\
\hline DVA & 61.8 & 6 & $9.7 \%$ \\
No associated DVA & 664.5 & 18 & $2.7 \%$ \\
\hline
\end{tabular}

* Rates are expressed per CM, not per patient. and to $3.3 \%$ after 5 years; however, only 11 hemorrhagic CMs had 5 years of untreated follow-up in this analysis. We noted trends for higher hemorrhage rates among CMs that were nonmultiple and those associated with DVAs. Brainstem CMs had a notably greater annual hemorrhage rate (16.7\% per lesion-year) than CMs in any other location, although the majority of these were initially hemorrhagic at presentation (14 [82\%] of 17). Permanent neurological morbidity, defined as a lasting neurological deficit at the last follow-up, was $29 \%$ per hemorrhage, increasing to $45 \%$ for brainstem, thalamic, or basal ganglia CM and decreasing to $15 \%$ for supratentorial lobar or cerebellar lesions. No mortality was associated with any $\mathrm{CM}$ hemorrhage in this series.

\section{Hemorrhage Risk Factors}

A summary of univariate and multivariate analyses of risk factors for hemorrhage is provided in Table 4. Brainstem location (HR 8.74, 95\% CI 3.73-20.5), prior hemorrhage (HR 8.30, 95\% CI 3.16-21.6), and solitary lesion (HR 4.88, 95\% CI 1.63-14.6) were statistically significant risk factors for hemorrhage on univariate analysis. Age, seizure presentation, patient sex, associated DVAs, and family history were not significant risk factors. After a multivariate analysis, prior hemorrhage (HR 4.63, 95\% CI 1.53-14.1), brainstem location (HR 4.42, 95\% CI 1.57-12.4), and associated radiologically apparent DVAs (HR 2.91, 95\% CI 1.04-8.09) remained statistically significant risk factors for hemorrhage (Fig. 1). In a post hoc univariate analysis, hemorrhage clustering within 3 years was statistically significant (HR 6.1, 95\% CI 1.72-21.7, p $=0.005)$ as compared with the hemorrhage rate after this initial period.

\section{Discussion}

Cerebral $\mathrm{CMs}$ are angiographically occult vascular malformations comprised of a collection of dilated sinusoidal vessels lined by a flattened endothelium without a reinforcing elastin or smooth muscle layer or a surrounding thin fibrous adventitia., $7,22,23$ The sinusoids contain blood of various ages and are directly apposed without intervening neural parenchyma in contradistinction to cerebral capillary telangiectasias. , $, 8,10,22$ Their natural course in the pediatric population remains relatively obscure, with only one recent study systematically evaluating their prevalence and natural course in children. ${ }^{2}$ The current analysis features the largest reported pediatric cohort with CMs. We excluded lesions smaller than $4 \mathrm{~mm}$ as a means of improving our study's reproducibility and external validity and reducing interobserver variability; however, this may exclude a cohort of relatively quiescent lesions and thus inflate our study's conclusions of lesion aggressiveness (seizure risk, hemorrhage risk). On the other hand, asymptomatic or mildly symptomatic events may have been missed.

Consistent with data in the adult literature, an overall imaging prevalence of $0.6 \%$ for CMs in children was reported in a recent study; ${ }^{2}$ however, the rate was lowest among infants $(0.2 \%)$ and increased with advancing age. In our study, we found no significant sex predilec- 
TABLE 4. Results of univariate and multivariate analysis of risk factors for hemorrhage

\begin{tabular}{|c|c|c|c|c|c|c|}
\hline \multirow[b]{2}{*}{ Factor } & \multicolumn{3}{|c|}{ Univariate } & \multicolumn{3}{|c|}{ Multivariate } \\
\hline & $\mathrm{HR}$ & $95 \% \mathrm{Cl}$ & $p$ Value & $\mathrm{HR}$ & $95 \% \mathrm{Cl}$ & $p$ Value \\
\hline Age presentation & 0.97 & $0.90-1.05$ & 0.459 & 0.99 & $0.91-1.07$ & 0.733 \\
\hline Female sex & 1.17 & $0.51-2.67$ & 0.705 & 0.74 & $0.29-1.86$ & 0.517 \\
\hline Seizure presentation & 1.02 & $0.37-2.76$ & 0.977 & 0.98 & $0.31-3.11$ & 0.973 \\
\hline Prior hemorrhage & 8.30 & $3.16-21.6$ & $1.64 \times 10^{-5}$ & 4.63 & $1.53-14.1$ & 0.007 \\
\hline Brainstem location & 8.74 & $3.73-20.5$ & $6.01 \times 10^{-7}$ & 4.42 & $1.57-12.4$ & 0.005 \\
\hline Associated DVA & 2.48 & $0.95-6.49$ & 0.064 & 2.91 & $1.04-8.09$ & 0.041 \\
\hline Solitary, nonmultiple & 4.88 & $1.63-14.6$ & 0.005 & 2.92 & $0.79-10.8$ & 0.108 \\
\hline Familial & 0.33 & $0.07-1.44$ & 0.141 & 1.63 & $0.28-9.5$ & 0.586 \\
\hline
\end{tabular}

tion among pediatric patients with CMs, consistent with a prior pediatric study ${ }^{2}$ as well as with a collective analysis of studies in adults. ${ }^{10}$ Although potentially influenced by referral patterns, our $10 \%$ rate of familial CM cases, as well as the $15 \%$ rate of patients with multiple CMs, was also consistent with a prior collective analysis of CMs in adults. ${ }^{10}$ Our analysis demonstrated that cerebellar CMs had greater proclivity for an association with radiologically apparent DVAs, a finding not previously reported. Although venous abnormalities have been implicated in the genesis of CMs, ${ }^{1,5,10}$ most studies, like our own, have demonstrated that radiologically apparent DVAs are seen in association with CMs in only $10 \%-20 \%$ of cases..$^{10,14,20}$ Though authors of early studies hypothesized increased lesion aggressiveness for CMs associated with DVAs, ${ }^{21}$ the presence of such lesions is infrequently evaluated as a risk factor for hemorrhage in natural history studies in adults..$^{10,12,14,20}$ However, in the one other study evaluating the natural course of CMs in pediatric patients, a notable trend toward a greater hemorrhage rate for CMs associated with DVAs was reported with a p value of $0.06 .{ }^{2}$ This finding was consistent with data in our study, potentially as a result of added statistical power, which revealed DVAs as a statistically significant risk factor for hemorrhage (HR $2.91,95 \%$ CI 1.04-8.09, $\mathrm{p}=0.04$ ).

Although we found the annual rate of de novo CM development to be $2.5 \%$ per lesion and $1.2 \%$ per patient, this rate was influenced by $\mathrm{CM}$ multiplicity and prior radiation. Patients with solitary CMs developed de novo lesions at a rate of $0.6 \%$ per lesion; this rate increased to $7.1 \%$ for patients with multiple CMs and to $2.6 \%$ for those with prior radiation.

Radiation-induced CM development was seen in 9\% of patients in this study, and notably none of these patients had radiologically apparent DVAs. Cavernous malformation development can occur after either conventional radiation $^{16}$ or stereotactic radiosurgery, ${ }^{15,17}$ though all patients with radiation-induced cerebral CMs in this study had undergone conventional fractionated radiation. A prior systematic review of 76 patients with radiation-induced CMs showed a mean patient age of 11.7 years with a latency of 8.9 years after radiation until $\mathrm{CM}$ detection, ${ }^{16}$ quite consistent with results seen in our study. Precluding a meaningful analysis of the natural history of radiation-induced CMs, all but 1 hemorrhagic, radiation-induced CM in our cohort was treated with early surgery.

\section{Hemorrhagic CMs}

Potentially influenced by referral bias, $62 \%$ of patients in this report presented with hemorrhagic CMs. As shown in this study and in several prior analyses, hemorrhage is a statistically significant risk factor for subsequent hemorrhage. $3,6,7,9,10,12$ In addition, our analysis was consistent with multiple prior adult and 1 prior pediatric study in demonstrating a $0.5 \%$ per lesion-year rate of hemorrhage for incidental CMs. ${ }^{2,3,9,10,12}$ We found the overall annual hemorrhage rate for hemorrhagic CMs to be $11.3 \%$; however, this rate was influenced by time from the initial hemorrhage, with statistically significant hemorrhage clustering occurring within 3 years of presentation. In a prior study by Barker et al., hemorrhage clustering was demonstrated within 2 years of presentation. ${ }^{6}$ This finding is an important one for both patient counseling and evaluating the efficacy of stereotactic radiosurgery for cerebral CMs. Most studies on the latter have shown a statistically significant decrease in the CM hemorrhage rate after 2 years;, $411,18,19$ it is unclear whether this rate, in fact, reflects the effect of radiosurgery or simply the natural history of the lesion. Given our findings, we recommend interval scanning at 3-6 months for hemorrhagic CMs deemed unsuitable for surgery; thereafter, these lesions can be followed annually. On the other hand, given the consistently low annual hemorrhage rate for incidental CMs in this study, including a $0.3 \%$ rate for a cohort with at least 5 years of follow-up, these lesions can be followed less frequently.

\section{Brainstem CMs}

Located in highly eloquent brain parenchyma, brainstem CMs have consistently demonstrated more aggressive, clinically apparent natural courses in both pediatric and adult studies. ${ }^{2,10,13,20}$ In our analysis, $82 \%$ of brainstem CMs presented with hemorrhage along with a subsequent annual hemorrhage rate of $16.7 \%$. While it is difficult to determine whether the high subsequent hemorrhage rate relates to the brainstem location or to the initial hemorrhagic presentation, this rehemorrhage rate is higher than that for hemorrhagic lesions presenting outside the brainstem. Another study dedicated to pediatric brainstem CMs reported a $79 \%$ rate of hemorrhagic presentation with a subsequent $11.7 \%$ annual hemorrhage rate. ${ }^{13}$ Interestingly, $38.8 \%$ of conservatively managed pediatric patients with brainstem CMs were clinically improved at follow-up, 
A
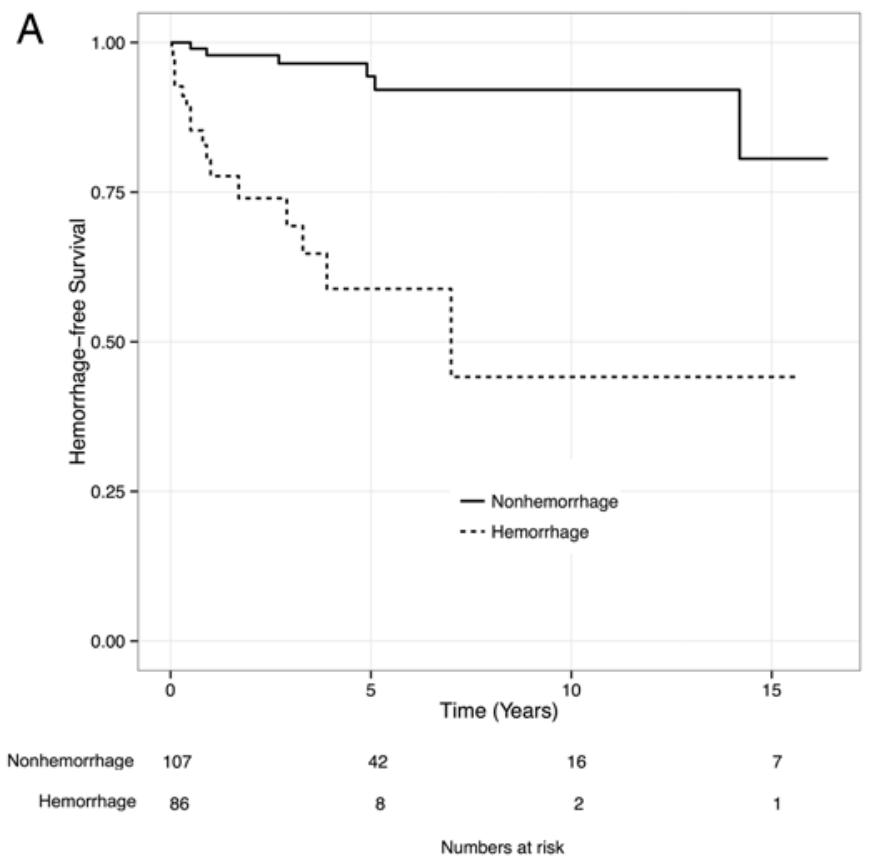

C

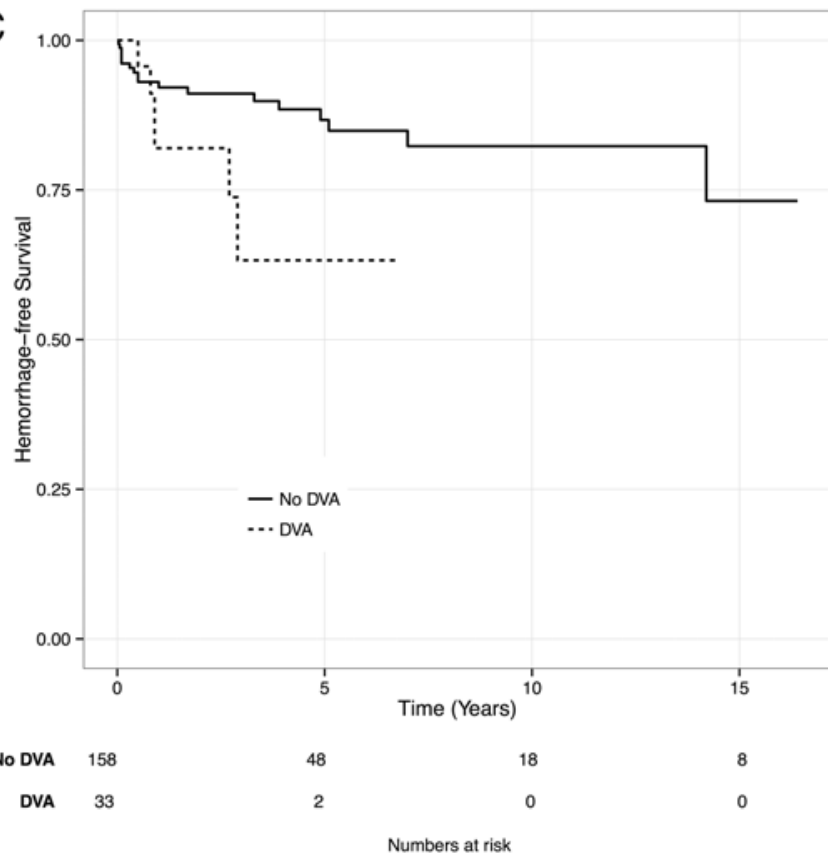

while $37.6 \%$ were unchanged and $23.5 \%$ were worse. In fact, $25 \%$ of patients attained complete recovery, a rate that increased to $49.2 \%$ for those with at least 5 years of follow-up. It is important to underscore that both our study and this subgroup analysis of pediatric brainstem CMs clearly illustrate early hemorrhage clustering for this subgroup of CMs in particular.

\section{Conclusions}

A prior hemorrhage, a brainstem location, and an associated DVA are significant risk factors for hemorrhage in children with CMs. Hemorrhage clustering within the

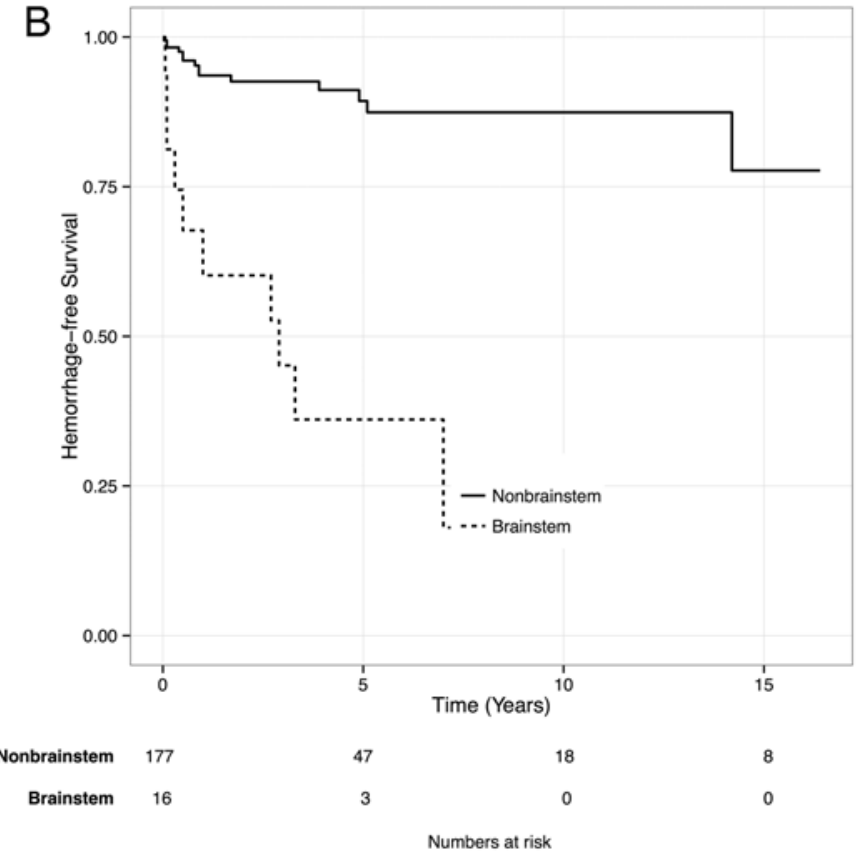

FIG. 1. Kaplan-Meier curves for hemorrhage-free survival without treatment in patients with hemorrhagic versus nonhemorrhagic presentation (A), those with brainstem versus nonbrainstem $\mathrm{CM}(\mathrm{B})$, and those with versus those without associated DVAs (C). Note the proclivity toward early hemorrhage clustering.

first 3 years of a bleed can occur, a factor to consider in patient counseling and when reviewing results of studies on $\mathrm{CM}$ radiosurgery.

\section{References}

1. Abdulrauf SI, Kaynar MY, Awad IA: A comparison of the clinical profile of cavernous malformations with and without associated venous malformations. Neurosurgery 44:41-47, 1999

2. Al-Holou WN, O’Lynnger TM, Pandey AS, Gemmete JJ, Thompson BG, Muraszko KM, et al: Natural history and imaging prevalence of cavernous malformations in children and young adults. J Neurosurg Pediatr 9:198-205, 2012 
3. Al-Shahi Salman R, Hall JM, Horne MA, Moultrie F, Josephson CB, Bhattacharya JJ, et al: Untreated clinical course of cerebral cavernous malformations: a prospective, populationbased cohort study. Lancet Neurol 11:217-224, 2012

4. Amin-Hanjani S, Ogilvy CS, Candia GJ, Lyons S, Chapman PH: Stereotactic radiosurgery for cavernous malformations: Kjellberg's experience with proton beam therapy in 98 cases at the Harvard Cyclotron. Neurosurgery 42:1229-1238, 1998

5. Awad IA, Robinson JR Jr, Mohanty S, Estes ML: Mixed vascular malformations of the brain: clinical and pathogenetic considerations. Neurosurgery 33:179-188, 1993

6. Barker FG II, Amin-Hanjani S, Butler WE, Lyons S, Ojemann RG, Chapman PH, et al: Temporal clustering of hemorrhages from untreated cavernous malformations of the central nervous system. Neurosurgery 49:15-25, 2001

7. Bertalanffy H, Benes L, Miyazawa T, Alberti O, Siegel AM, Sure U: Cerebral cavernomas in the adult. Review of the literature and analysis of 72 surgically treated patients. Neurosurg Rev 25:1-55, 2002

8. Clatterbuck RE, Moriarity JL, Elmaci I, Lee RR, Breiter SN, Rigamonti D: Dynamic nature of cavernous malformations: a prospective magnetic resonance imaging study with volumetric analysis. J Neurosurg 93:981-986, 2000

9. Flemming KD, Link MJ, Christianson TJ, Brown RD Jr: Prospective hemorrhage risk of intracerebral cavernous malformations. Neurology 78:632-636, 2012

10. Gross BA, Lin N, Du R, Day AL: The natural history of intracranial cavernous malformations. Neurosurg Focus 30(6):E24, 2011

11. Karlsson B, Kihlström L, Lindquist C, Ericson K, Steiner L: Radiosurgery for cavernous malformations. J Neurosurg 88:293-297, 1998

12. Kondziolka D, Lunsford LD, Kestle JR: The natural history of cerebral cavernous malformations. J Neurosurg 83:820 824,1995

13. Li D, Hao SY, Tang J, Xiao XR, Jia GJ, Wu Z, et al: Clinical course of untreated pediatric brainstem cavernous malformations: hemorrhage risk and functional recovery. J Neurosurg Pediatr 13:471-483, 2014

14. Moriarity JL, Wetzel M, Clatterbuck RE, Javedan S, Sheppard JM, Hoenig-Rigamonti K, et al: The natural history of cavernous malformations: a prospective study of 68 patients. Neurosurgery 44:1166-1173, 1999

15. Motegi H, Kuroda S, Ishii N, Aoyama H, Terae S, Shirato $\mathrm{H}$, et al: De novo formation of cavernoma after radiosurgery for adult cerebral arteriovenous malformation-case report. Neurol Med Chir (Tokyo) 48:397-400, 2008
16. Nimjee SM, Powers CJ, Bulsara KR: Review of the literature on de novo formation of cavernous malformations of the central nervous system after radiation therapy. Neurosurg Focus 21(1):e4, 2006

17. Park YS, Kim SH, Chang JH, Chang JW, Park YG: Radiosurgery for radiosurgery-induced cavernous malformation. World Neurosurg 75:94-98, 2011

18. Pham M, Gross BA, Bendok BR, Awad IA, Batjer HH: Radiosurgery for angiographically occult vascular malformations. Neurosurg Focus 26(5):E16, 2009

19. Pollock BE, Garces YI, Stafford SL, Foote RL, Schomberg PJ, Link MJ: Stereotactic radiosurgery for cavernous malformations. J Neurosurg 93:987-991, 2000

20. Porter PJ, Willinsky RA, Harper W, Wallace MC: Cerebral cavernous malformations: natural history and prognosis after clinical deterioration with or without hemorrhage. J Neurosurg 87:190-197, 1997

21. Pozzati E, Acciarri N, Tognetti F, Marliani F, Giangaspero F: Growth, subsequent bleeding, and de novo appearance of cerebral cavernous angiomas. Neurosurgery 38:662-670, 1996

22. Robinson JR, Awad IA, Little JR: Natural history of the cavernous angioma. J Neurosurg 75:709-714, 1991

23. Scott RM, Barnes P, Kupsky W, Adelman LS: Cavernous angiomas of the central nervous system in children. J Neurosurg 76:38-46, 1992

\section{Disclosure}

The authors report no conflict of interest concerning the materials or methods used in this study or the findings specified in this paper.

\section{Author Contributions}

Conception and design: Gross, Scott, Smith. Acquisition of data: Gross. Analysis and interpretation of data: Gross, Du, Orbach. Drafting the article: Gross. Critically revising the article: Du, Orbach, Scott, Smith. Reviewed submitted version of manuscript: all authors. Approved the final version of the manuscript on behalf of all authors: Gross. Statistical analysis: Gross. Du, Administrative/technical/material support: Scott, Smith. Study supervision: Scott, Smith.

\section{Correspondence}

Bradley A. Gross, Department of Neurological Surgery, Boston Children's Hospital and Harvard Medical School, 300 Longwood Ave., Boston, MA 02115. email: bgross1@ partners.org. 